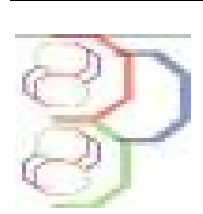

\title{
Essai de phytostabilisation des sites contaminés par les éléments traces métalliques dans la région de Lubumbashi (RD. Congo) : Gamme de tolérance de Jatropha curcas au sulfate de cuivre.
}

\author{
Mununga Katebe Félicien ${ }^{a^{*}}$, Kaumbu Kyalamakasa Jean-Marca, Chuimika Mulumbati Magnifiquea, \\ Mwilambwe Kapoma Xaviera, Maloba Kazembe Jean-Pierrec, Banza llunga Meschackc, Bushabu \\ Mingashangaa, Mpundu Mubemba Mulambi Michela的 \\ a Département de phytotechnie, Faculté des Sciences Agronomiques, Université de Lubumbashi, BP 1825 Lubumbashi, \\ RD Congo ; \\ bÉcole d'Hôtellerie et Tourisme. BP 1825, Université de Lubumbashi, BP 1825 Lubumbashi, RD Congo ; \\ ${ }^{\circ}$ Centre de Recherche Agro-alimentaire(CRAA) Lubumbashi, RD Congo. \\ *Auteur correspondant : munungakatebe@gmail.com , +243815778141
}

Original submitted in on $1^{\text {st }}$ February 2017. Published online at www.m.elewa.org on 30th April 2017 https://dx.doi.org/10.4314/jab.v112i1.13

\section{RESUME}

Problématique : Les activités industrielles notamment la métallurgie dans la ville de Lubumbashi, ont engendré via leurs rejets atmosphériques (canalisés et diffus) une forte contamination des sols aux alentours des usines. Cependant, les sols à usages divers (forestiers, agricoles ou résidentiels), leur pollution par les éléments traces métalliques (ETM) constitue un danger plus ou moins sévère pour l'environnement et pour les populations. Objectif : Cette étude a été menée pour évaluer la croissance et le potentiel de phytostabilisation de Jatropha curcas installé sur un substrat contaminé à différentes doses de sulfate de cuivre.

Méthodologie et résultats: Deux essais ont été conduits simultanément au champ expérimental de la faculté des Sciences Agronomiques de l'Université de Lubumbashi (FSA/Unilu), suivant un dispositif complètement randomisé comprenant 4 répétitions de 7 traitements constitués par des doses croissantes de sulfate de cuivre apportées artificiellement au sol normal sous forme de poudre. Les résultats relatifs aux paramètres végétatifs notamment le taux de reprise, le taux de levée, le taux de survie, la taille et le diamètre au collet des plantes ont été soumis à l'analyse de la variance (ANOVA) et au test de Tukey. Les résultats d'analyse chimique dans les sols et dans les plantes ont été comparés aux normes internationales. Les résultats obtenus montrent que Jatropha curcas s'est montré vigoureux sur les traitements ayant reçu des faibles doses de sulfate de cuivre

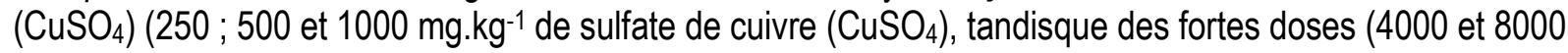
mg. $\mathrm{kg}^{-1}$ de sulfate de cuivre ( $\mathrm{CuSO}_{4}$ ) ont induit des mortalités maximales des plantules (100\%). Par ailleurs, les calculs de bioaccumulation et translocation montrent des taux élevés de bioaccumulation du sulfate de cuivre de Cuivre dans les racines et des faibles taux de translocation en sulfate de cuivre de Cuivre des plantes de Jatropha curcas vers les parties aériennes.

Conclusion et application des résultats : La phytostabilisation des sols contaminés en ETMs avec l'espèce Jatropha curcas est possible dans la région de Lubumbashi, du faite que cette espèce est tolérante jusqu'à des 
doses allant jusqu'à 2000 ppm par semis indirecte et minimise les risques de transfert d'ETM vers les autres compartiments de l'environnement.

Mots-clés : Phytostabilisation, Jatropha curcas, ETMs, Lubumbashi.

\section{ABSTRACT}

Problem: Industrial activities, especially in the sector of metallurgy in the city of Lubumbashi, caused a high contamination of the soil around the factories via their atmospheric discharges (channelled and diffuse). However, contaminated soils used for various purposes in forestry, agricultural or residential areas, has negative impact in their surrounding; increasing pollution by adding several amount of heavy metals, constitute an important source of damage for its immediate environment and nearby populations.

Objectives: This study was conducted to evaluate the growth and phytostabilisation potential using Jatropha curcas on a contaminated substrate at different doses of copper sulphate.

Methodology and results: The experiment was conducted at the experimental field of the Faculty of Agricultural Sciences, University of Lubumbashi, following a completely randomized system comprising 4 repetitions of 7 treatments. The determination of the tolerance range was subjected to variance analysis (ANOVA), while the other vegetative parameters were subjected to the multiple comparison test (Tukey test). Thus, the chemical composition of the various treatments was analyzed to deduce the tolerance threshold of Jatropha curcas to cupric contamination. Results indicated that Jatropha curcas was vigorous on treatments with low doses of copper sulphate (250, 500 and $1000 \mathrm{mg} . \mathrm{kg}-1$ CuSO4), while high doses of copper sulphate induced of seedling mortality (2000, 4000 and T6 $=8000$ mg.kg-1 CuSO4), except for only transplanted individuals which had intermediate results for the treatment contaminated with (2000 mg.kg-1 CuSO4), unlike to seeded individuals. Furthermore, bioaccumulation and translocation calculations display high levels of copper accumulated in the tissues of the roots due to the soil content and to the low level of $\mathrm{Cu}$, translocation gives Jatropha curcas the power to minimize the rate of passage towards the aerial parts.

Conclusion and application of results: the phytostabilisation of soils polluted by the use of heavy metals is an effective and less costly solution, thus the use of Jatropha curcas would be an alternative to achieve the goal being given that can tolerate concentrations of copper itself (Up to 2000 ppm when using transplanting), thusly the domestication of this species and his botanical knowledge is interesting.

Keywords : Phytostabilisation, phytotranslocation, biometric, Jatropha curcas

\section{INTRODUCTION}

L'essor de la ville de Lubumbashi (RD Congo) est depuis sa création intimement lié à l'extraction minière et au traitement des minerais du cuivre $(\mathrm{Cu})$ et cobalt (Co). Ces activités connaissent un développement considérable dans tout le Haut Katanga depuis quelques années. Ces usines d'extraction de minerais sont nombreuses et la densité de la population qui est en perpétuelle augmentation, ce qui ne va pas sans poser des questions d'ordre sanitaire (Mpundu et al., 2014). Les activités minières, notamment la métallurgie, dans la province du Haut-Katanga ont engendré via leurs rejets atmosphériques (canalisés et diffus), une forte contamination des sols aux alentours des usines. C'est ainsi que l'usage de ces sols (forestiers, agricoles ou résidentiels), pollués par les éléments traces métalliques (ETM) constitue un danger plus ou moins sévère pour l'environnement et pour les populations (Francis, 2012). Dans la ville de Lubumbashi, il a été constaté que le secteur situé sous les vents alizés du Sud- est présente des sols riches en $\mathrm{Cu}$ et autres métaux lourds, par les retombées de poussières métallifères. Cette situation a favorisé le remplacement de la forêt claire originelle de Miombo, par une steppe courte (Malaisse, 1997) ainsi que de vastes zones de sol dénudé. Toutefois, le sol resté nu devient l'objet d'une érosion hydrique et éolienne soutenue. Cependant, pour la diminution des risques de dispersion de la contamination inhérents aux dépôts de résidus miniers, la phytostabilisation pourrait être la solution; c'est ainsi cette stratégie 
d'assainissement peut être utilisée même à des niveaux de contamination élevés d'ETM dans les substrats (El himer et al., 2013). La valorisation des sols dénudés par le reboisement, l'identification et le choix de plantes appropriées, tolérantes aux ETM pour l'assainissement des terrains des sols contaminés sans affecter l'équilibre de l'écosystème sont une tâche laborieuse. L'espèce Jatropha curcas L., arbuste pérenne, résistante à la sècheresse et dont les graines constituent une matière première intéressante pour l'obtention d'une huile à haute qualité combustible (Achten et al., 2008), est considérée comme une des solutions possibles à la crise énergétique qui menace le monde suite à l'augmentation structurelle du prix des hydrocarbures. Cet intérêt repose principalement sur la capacité de produire des graines de façon rentable dans des conditions de culture difficiles sur des terres incultes, pauvres, marginales où il n'existe aucune possibilité d'irrigation et sans application d'intrants (Francis et al., 2005 ; Brittaine et Lutaladio, 2010 ; Ahoton et al., 2011). Des études sur les problèmes des sols contaminés et leur remédiation par J. curcas (Qihanget al., 2011 ; Jamil et al., 2009 ;

\section{MATERIEL ET METHODES}

Milieu d'étude : cet essai a été installé à Lubumbashi (RD Congo), chef lieu de la province du Haut-Katanga localisée à $11^{\circ} 61^{\prime}$ Sud et $27^{\circ} 48^{\prime}$ Est, et à $1257 \mathrm{~m}$ d'altitude. La ville de Lubumbashi est caractérisée par un climat du type Cw6 de la classification de Koppën (FAO, 2005). Cette dernière présente une température moyenne annuelle de $20^{\circ} \mathrm{C}$ (Mujinya et al., 2011). Ainsi la saison sèche s'étend de Mai à Septembre, et Octobre est un mois de transition. Par contre la saison de pluie
Yadavet al., 2009, 2010 ; Leyeet al., 2009 ; Mangkoedihardjo et al., 2008 ; Juwarkar et al., 2007 (a et b), Kumar et al., 2007) ont donné des résultats intéressants dans le but d'améliorer la croissance de cette plante. Les résultats obtenus sur les études similaires menées dans la région de Lubumbashi ont révélé les possibilités de phytostabilisation et de réduction de la mobilité des ETM dans les sols contaminés par les espèces herbacées (Shutcha et al., 2014 ; Mpundu et al., 2013). Cependant, pour une amélioration du couvert végétal, l'utilisation des plantes ligneuses dans la remédiation des sols contaminés s'avère impérieux dans cette région. Outre son utilisation dans la phytostabilisation, J. curcas intervient dans la production de biogaz (Biocarburant de première génération) (Pellet et al.,2007 ; Neffet al., 2008),dont son huile constitue une solution à la flambée des prix des hydrocarbures. C'est dans cette perspective que ce travail a été initié pour évaluer la croissance et le potentiel de phytostabilisation de J. curcas sur un substrat contaminé à des doses croissantes de sulfate de cuivre $\left(\mathrm{CuSO}_{4}\right)$.

commence de Novembre et se termine à mi-avril (Bruneau., 1983).

Sols : La couche de surface des sols $(0-25 \mathrm{~cm})$ du champ expérimental de la FSA/Unilu a été utilisée comme substrat d'étude. Ce matériel est un ferralsol de la série Katuba, selon la classification de I'INEAC (Mpundu, 2010), et dont l'analyse morphologique a révélé une texture argilo-sableuse. Les résultats d'analyse des sols du champ expérimental de la FSA/Unilu sont repris dans le (tableau 1).

Tableau 1. Caractéristiques chimiques de sol du champ expérimental de la FSA/Unilu

\begin{tabular}{l|l|l|l}
\hline $\mathrm{pH}$ & $\mathrm{Cu}(\mathbf{p p m})$ & Norme \\
\hline $\mathrm{pH}$ eau & $\mathrm{pH} \mathrm{KCl}$ & \multirow{2}{*}{148} & 30 \\
\hline 6.19 & 4.25 & 148 & 30 \\
\hline
\end{tabular}

Plante test : Les graines de Jatropha curcas L. utilisées comme semences pour les semis direct et indirect dans cette expérimentation ont été obtenues sur les capsules des arbustes trouvés au quartier Gambela III, de la commune Lubumbashi (Fig. 1). 


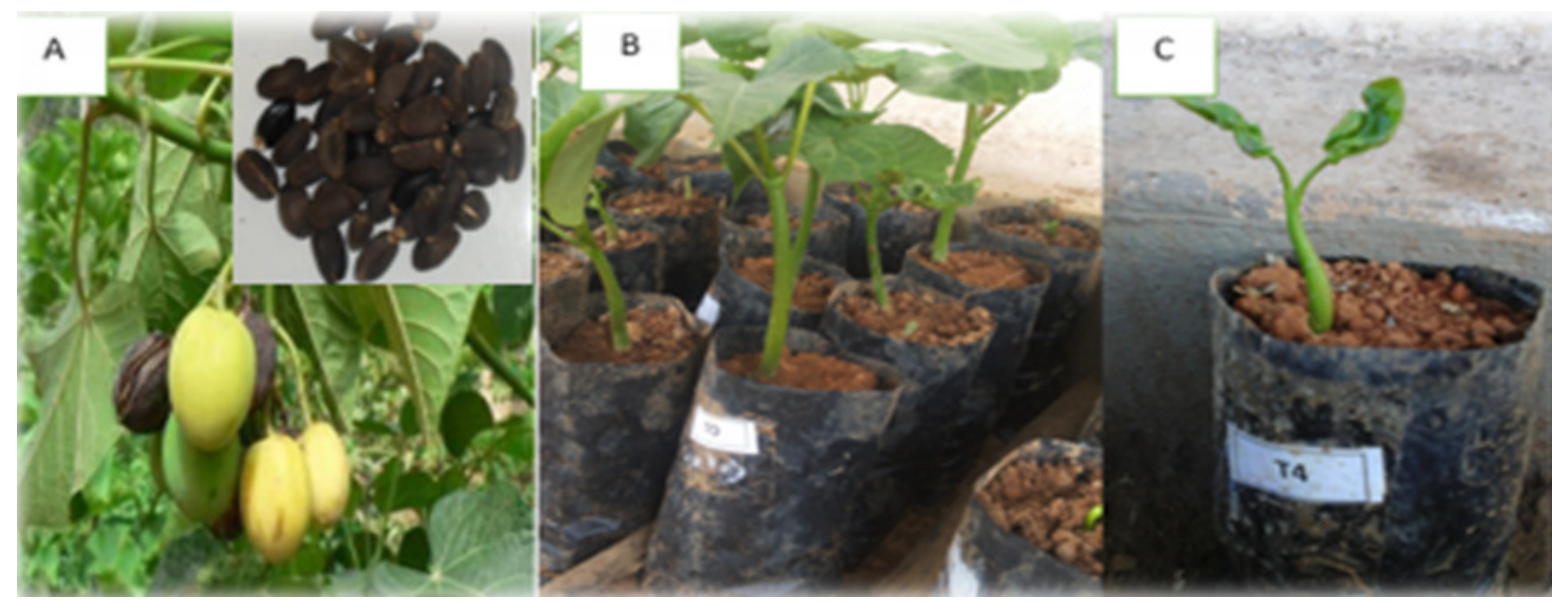

Figure 1. Photo de graines et plantules de Jatropha curcas L. (Crédit : Mununga Katebe Félicien).

Conduite de l'essai : 2 essais (semis direct de graines et plantules de $J$. curcas) ont été conduit parallèlement en sachets de polyéthylènes, remplis respectivement de $2 \mathrm{~kg}$ et $4 \mathrm{~kg}$ de sol. Les sols ont été séchés à l'air libre pendant 4 jours avant la contamination artificielle en $\mathrm{Cu}$. Les différentes doses du Cu ont été obtenues par l'apport du sulfate du Cu au sol sec du champ expérimental de la FSA/Unilu dans un bassin plastique. Les substrats bien mélangés (en fonction des doses retenues) ont été placés dans des sachets et suivis des arrosages pendant 7 jours avant semis direct ou plantation. Ces sachets ont été placés derrière l'auditoire de Bac 1 Agronomie générale de la FSA/Unilu. Le semis direct a été effectué à raison d'une graine par sachet de $2 \mathrm{~kg}$. Parallèlement, les plantules de 3 feuilles ont été choisies pour la transplantation 7 jours après semis en pépinière à raison d'une plantule par sachet de $4 \mathrm{~kg}$ de sol. La fréquence d'arrosage a été d'une fois par jour aux doses respectives de 75 et $150 \mathrm{ml}$ d'eau par sachet de 2 et $4 \mathrm{~kg}$. Le binage a été effectué à la fréquence d'une fois pour 15 jours, en vue de favoriser l'infiltration de l'eau.

Paramètres observés et analyse des échantillons :Les taux de levée et de reprise ont été observés respectivement 7 jours après semis et transplantation. La survie, la taille des plantes, le nombre des feuilles, ainsi que le diamètre au collet ont été observés à un écart de 15 jours; pendant 3 mois. Les analyses du sol du champ expérimental, la détermination du $\mathrm{pH}\left(\mathrm{H}_{2} \mathrm{O}, \mathrm{KCl}\right)$ ainsi que les variables analytiques (sols et végétaux) ont été analysées au Laboratoire Agro-pédologique, Science du sol de la FSA/Unilu en RD Congo. Par ailleurs les teneurs totales en $\mathrm{Cu}$ dans les plantes ont été analysées par méthode de spectrométrie à fluorescence à rayon $\mathrm{X}$ à l'aide d'un appareil de Marc Olympus. L'indice biométrique a été estimé sur base du calcul des facteurs de transfert (facteur de bioaccumulation: Ft/b) et de translocation (Ft) (Bidar et al.,2007; Marchiol et al., 2004).

$$
F t / b=\frac{\text { Concentrationdumétaldanslesracinesdelaplantes }(\mathrm{mg} / \mathrm{kg})}{\text { Concentrationdumétaldanslesol }(\mathrm{mg} / \mathrm{kg})}
$$

$$
t=\frac{\text { Concentrationdumétaldanslesfeullesdelaplantes }(\mathrm{mg} / \mathrm{kg})}{\text { Concentrationdumétaldanslesracinesdelaplante }(\mathrm{mg} / \mathrm{kg})}
$$

Les analyses statistiques ont été effectuées à l'aide du logiciel Minitab version 16. L'analyse de la variance et le test de Tukey ont été utilisés pour comparer les différentes moyennes. Les résultats d'analyse des échantillons des sols et des végétaux ont été confrontés aux normes internationales, sachant que les valeurs seuils en ETM extraits à l'EDTA pour la plupart d'ETMs sont moins disponibles. Les résultats obtenus ont été comparés aux seuils de toxicité en ETMs totaux, quand bien même la teneur totale n'explique pas totalement l'exposition des végétaux aux ETMs (Mench\&Baize, 2004). 


\section{RESULTATS}

Réaction du sol : Les substrats expérimentaux utilisés dans cet essai ont été classées dans les sols acides (Baize, 2004).Cependant, les sols ayant reçu les doses massives au-delà de 500 ppm de Cu ont montré des valeurs pouvant être classées dans la gamme de très acide.

Tableau 3 : Caractéristiques chimiques des sols de différents traitements.

\begin{tabular}{c|c|c|c}
\hline Échantillon & $\mathbf{p H ~ e a u}$ & $\mathbf{p H ~ K C l}$ & $\Delta \mathbf{p H}$ \\
\hline $\mathrm{T} 0$ & 6,33 & 6,25 & 0,08 \\
\hline $\mathrm{T} 1$ & 6,6 & 6,0 & 0,1 \\
\hline $\mathrm{T} 2$ & 6,1 & 6,0 & 0,4 \\
\hline $\mathrm{T} 3$ & 5,7 & 5,3 & 0,4 \\
\hline $\mathrm{T} 4$ & 5,4 & 5,4 & 0,0 \\
\hline $\mathrm{T} 5$ & 5,3 & 5,2 & 0,1 \\
\hline $\mathrm{T} 6$ & 5,2 & 5,1 & 0,1 \\
\hline
\end{tabular}

Paramètres végétatifs

Influence de sulfate de cuivre sur le comportement de Jatropha curcas

Levée et reprise de Jatropha curcas : La levée (à gauche) et la reprise (à droite) de J. curcas ont été effectives pour tous les traitements considérés (Figure 1.). Quelques soient les variations, la comparaison des moyennes par le test de TUKEY a révélé qu'il n'existe aucune différence significative $(P<0,05)$ entre les moyennes des taux de levée ainsi que de reprise de plantules de J. curcas; Autrement dit, les différentes doses du Cu n'ont pas influencé la levée ainsi que la reprise de J. curcas.

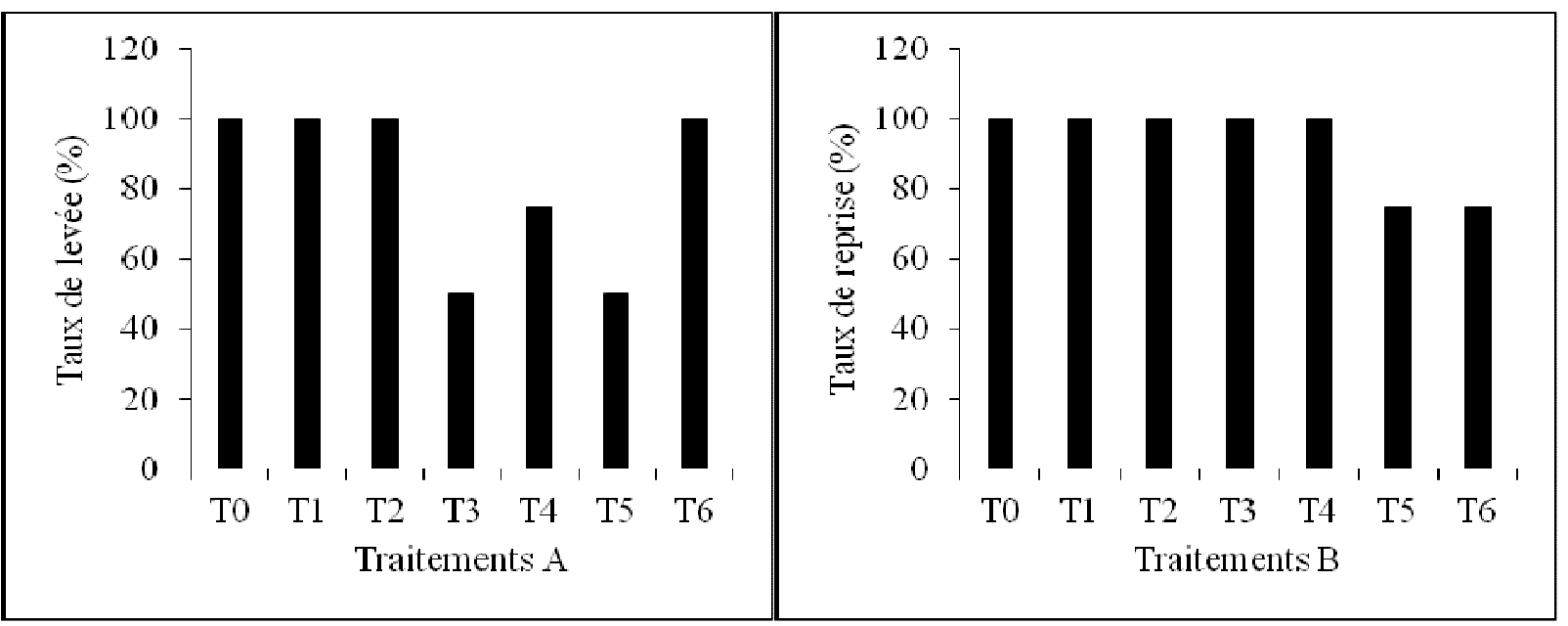

Figure 1 : Taux de levée et de reprise de $\mathrm{J}$. curcas en fonction de doses de sulfate de Cuivre $\left(\mathrm{CuSO}_{4}\right)$. T0 : Témoin ; T1 :

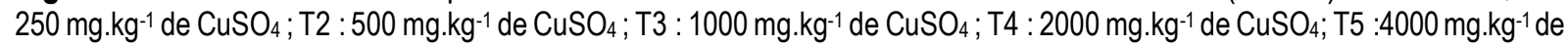
$\mathrm{CuSO}_{4} ; \mathrm{T} 6: 8000 \mathrm{mg}^{\prime} \mathrm{kg}^{-1} \mathrm{de} \mathrm{CuSO}_{4}$.

Survie de Jatropha curcas : L'analyse de la variance (ANOVA) indique qu'il existe des différences significatives entre les traitements $(p=0,000)$ pour le paramètre survie. La survie a été maximale (100\%) pour les plantules de $\mathrm{J}$. curcas obtenues par semis sur les faibles doses (T1 et T2) et similaire ( $p>0.05)$ au témoin (Figure 2 à gauche). Par contre, la mortalité a été maximale sur les doses massives allant de 2000 (T4) à8000 mg. $\mathrm{kg}^{-1}$ de sulfate de cuivre $\left(\mathrm{CuSO}_{4}\right)(\mathrm{T} 6)$. Le taux de survie a été réduit de moitié à partir de la dose T3. Par ailleurs, les taux de survie obtenus avec les individus repiqués sur les traitements T1, T2 et T3ont été similaires au témoin T0. La survie des plantes repiquées a été réduite de moitié à la dose de T4, au moment où la mortalité a été maximale avec les doses massives (T5 et T6) (Figure 2 à droite). 

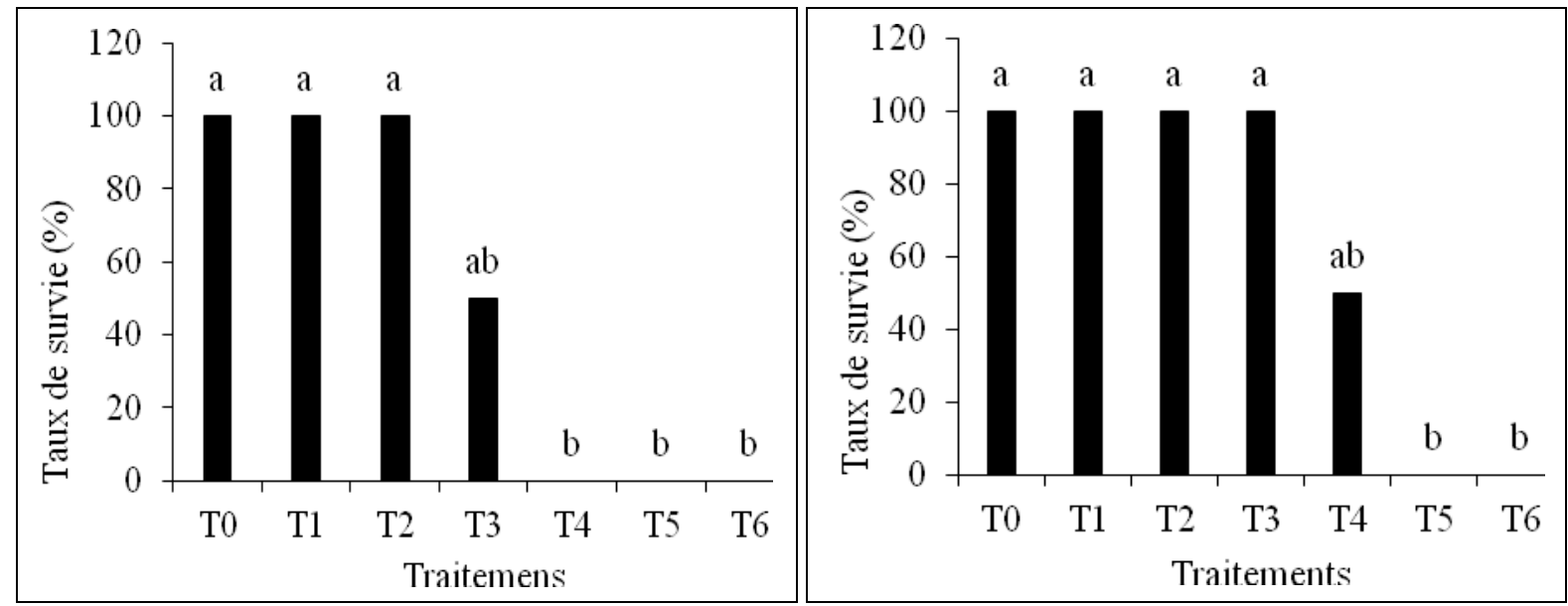

Figure 2. Taux de survie à 90 jours après levée et reprise (T0 : témoin $0 \mathrm{ppm}$ de $\mathrm{CuSO}_{4} ; \mathrm{T} 1: 250 \mathrm{mg}^{\mathrm{kg}}{ }^{-1} \mathrm{de} \mathrm{CuSO}_{4} ; \mathrm{T} 2: 500$

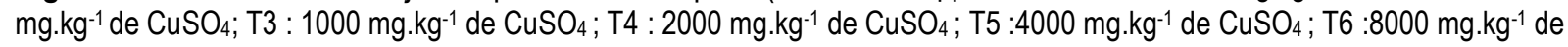
$\mathrm{CuSO}_{4}$.)

Nombre des feuilles : L'analyse de la variance montre qu'il existe des différences significatives $(p=0,000)$ entre les traitements pour le nombre de feuilles (figure 3). La comparaison des moyennes des feuilles par le test de Tukey montre que le nombre de feuilles a diminué avec la dose de $\mathrm{Cu}$ à partir de $\mathrm{T} 4$. Le nombre des feuilles obtenues sur les faibles doses (T1, T2 et T3) ont été similaires au témoin. Par contre, à partir de la dose $\mathrm{T} 5$, le nombre de feuilles par plante de $J$. curcas a été inférieur par rapport au témoin TO pour les deux essais.
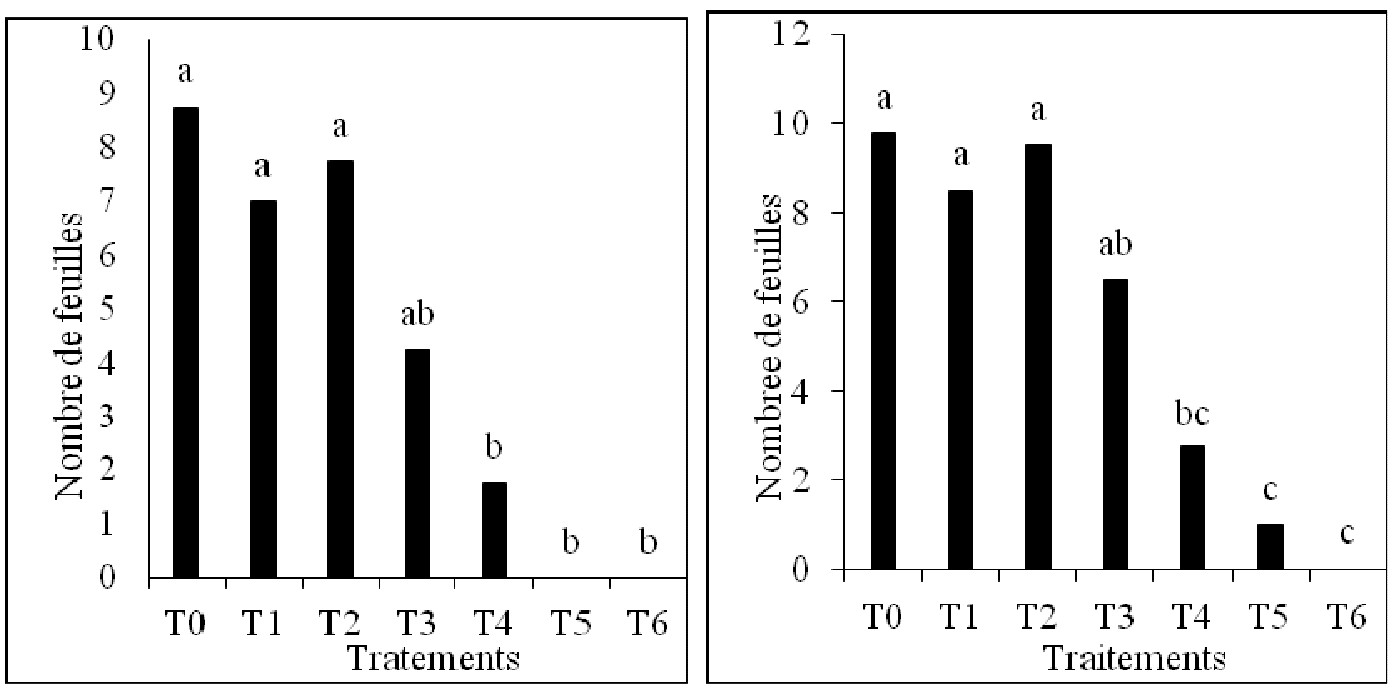

Figure 3 : Nombre de feuilles de plants de $J$. curcas selon les traitements. T0 : témoin 0 ppm de $\mathrm{CuSO}_{4} ; \mathrm{T1}: 250$

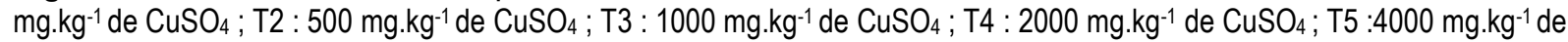
$\mathrm{CuSO}_{4} ; \mathrm{T} 6: 8000{\mathrm{mg} . \mathrm{kg}^{-1}}^{-1} \mathrm{CuSO}_{4}$.

Hauteur des plants : L'analyse de variance montre qu'il existe des différences significatives entre les traitements $(p=0.000)$, pour le paramètre Hauteur des plants (Figure.4). II ressort de la comparaison des moyennes par le test de Tukey que, les doses minimales T1, T2 et T3 ont donné les moyennes de taille similaires au témoin, contrairement aux doses massives de $\mathrm{CuSO}_{4}$ (T5 et T6). 


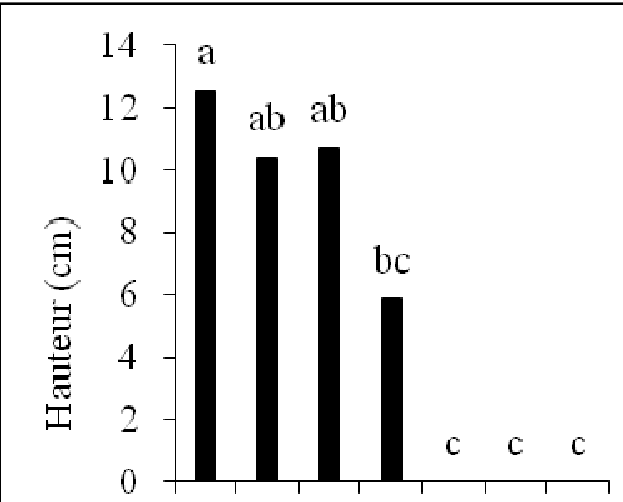

T0 $\mathrm{T} 1 \mathrm{~T} 2 \mathrm{~T} 3 \mathrm{~T} 4 \mathrm{~T} 5 \mathrm{~T} 6$

Traitements

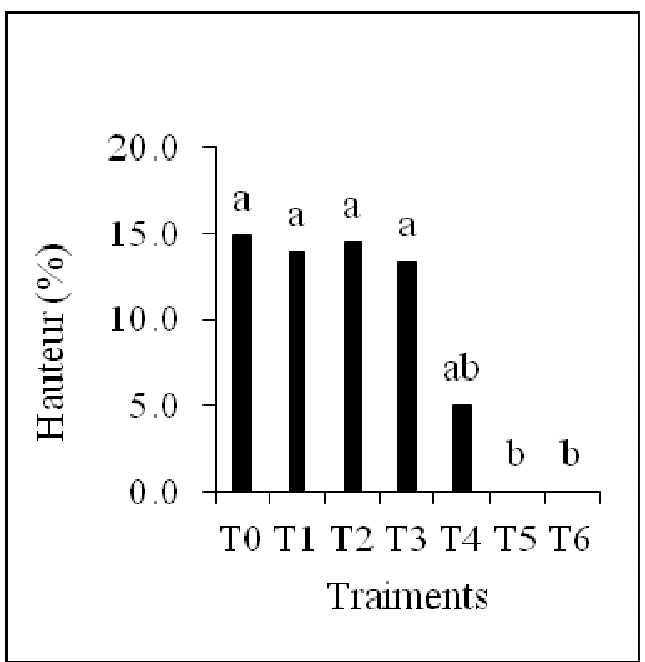

Figure 4 : Hauteur des plants de $\mathrm{J}$. curcas selon les traitements. T0 : témoin $0 \mathrm{ppm}$ de $\mathrm{CuSO}_{4} ; \mathrm{T}^{1}: 250 \mathrm{mg}^{\mathrm{kg}}{ }^{-1} \mathrm{de}_{\mathrm{CuSO}}$;

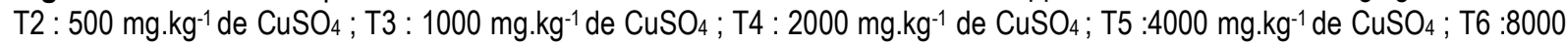

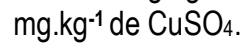

\section{Indice biométrique}

Bioaccumulation du Cuivre : Les teneurs en Cu observées dans les parties aériennes et racinaires de J. curcas ont excédées largement les normes établies par Kabata-Pendias\&Pendias (1992) (tableau 3). Les racines ont montré les teneurs plus élevées en $\mathrm{Cu}$ que les feuilles. La teneur en Cu observée dans les organes de J. curcas décroît avec le traitement appliqué. Le calcul du facteur de bioconcentration montre que le $\mathrm{Cu}$ est plus absorbé au niveau des racines des plantes installées sur différents traitements et cela indépendamment des concentrations appliquées (figure 8).

Tableau 4. Teneurs moyennes en Cu dans les racines et feuilles de J. curcas

\begin{tabular}{c|c|c|c|c|c}
\hline Échantillon & Racine & Feuille & Norme (ppm) & Référence \\
\hline T0 & 138 & 133 & & \\
\hline T1 & 345 & 111 & \multirow{2}{*}{$5-20$} & \multirow{2}{*}{ Kabata Pendias (1992) } \\
\hline T2 & 425 & 135 & & & \\
\hline T3 & 998 & 157 & & & \\
\hline
\end{tabular}

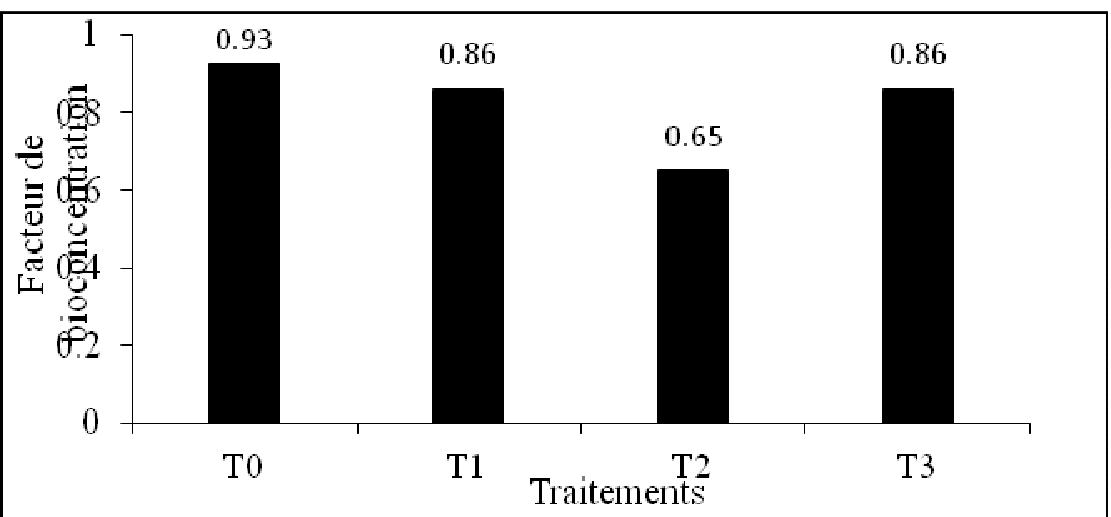

Figure 3 : Facteur de bioconcentration du Cu dans les racines de J. curcas. T0 : témoin Oppm de $\mathrm{CuSO}_{4} ; \mathrm{T} 1: 250$ mg.kg-1 de $\mathrm{CuSO}_{4} ; \mathrm{T}^{2}: 500{\mathrm{mg} . \mathrm{kg}^{-1} \mathrm{de} \mathrm{CuSO}_{4} ; \mathrm{T} 3: 1000 \mathrm{mg}^{\mathrm{kg}}{ }^{-1} \text { de CuSO}}_{4}$. 
Parallèlement, le calcul du facteur de translocation (figure 9) révèle que les traitements appliqués (T1, T2 et T3) se sont caractérisés par un faible facteur de translocation en
Cu par rapport au témoin (TO). Autrement dit, le facteur de translocation de J. curcas décroit avec la teneur en $\mathrm{Cu}$ dans le sol.

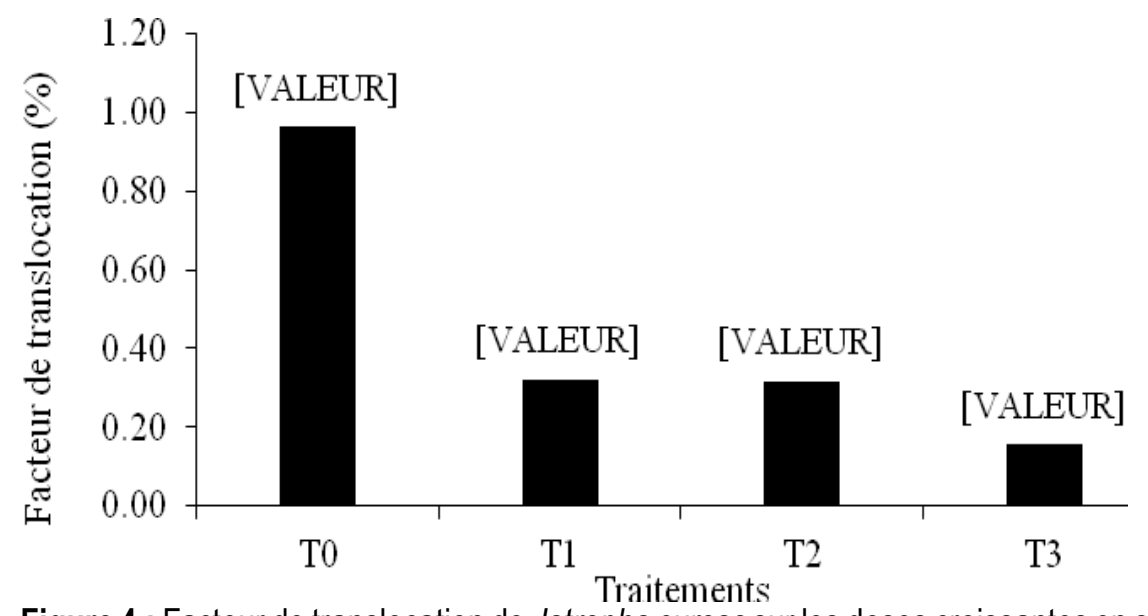

Figure 4 : Facteur de translocation de Jatropha curcas sur les doses croissantes en sulfate de Cuivre T0 : témoin Oppm de

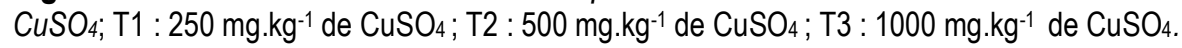

\section{DISCUSSION}

Après expérimentation les substrats expérimentaux où a poussés le Jatropha curcas ont été classés dans la catégorie des sols acides (Baize, 2004). La valeur du pH obtenu dans le sol témoin (TO) de la présente expérimentation est légèrement acide, contrairement à la neutralité observée sur les mêmes sols du champ expérimental de FSA/Unilu dans les études précédentes effectuées par Useni et al. (2014) et Ngoy (2007). Cette différence pourrait être attribuée à l'hétérogénéité du sol du champ expérimental de la FSA/Unilu. Gaston et al. (2001) soulignent que l'existence d'une variabilité des propriétés physico-chimiques à l'échelle métrique et plurimétrique. Parallèlement, les substrats ayant reçu les doses massives de sulfate du $\mathrm{Cu}$ (> 500 ppm) ont été classées dans la catégorie des sols très acides. L'apport des doses excessives du sulfate pourrait expliquer cette forte acidification des substrats utilisés comme traitements. En présence de l'eau, le sulfate donne l'acide sulfurique pouvant acidifier les sols. Par ailleurs, il a été observé que la teneur en Cu dans le sol témoin (148 ppm) a excédé légèrement la norme standard (Bowen,1979). Cette tendance à la hausse de la teneur en $\mathrm{Cu}$ dans les sols du champ expérimental de la FSA/Unilu pourrait se justifier par la contamination diffuse attribuée principalement à la proximité des essais de phytorémediation ex situ, des sols contaminés en ETMs, du lieu de prélèvement de sol. Zang et al. (2003) rapportent l'extension des niveaux de contamination plus faibles des sols sur plusieurs dizaines de kilomètres, suite à une pollution diffuse. Les résultats obtenus sur la croissance végétative ont indiqué une nette influence de doses croissantes de sulfate de cuivre $\left(\mathrm{CuSO}_{4}\right)$, à l'exception de la levée et la reprise de J. curcas. Les traitements ayant reçu des doses minimales allant de 250 à $1000 \mathrm{ppm}$ de sulfate de Cu ont montré des résultats similaires par rapport au témoin (T0) sur l'ensemble de paramètres observés. Par contre, les traitements contaminés avec des doses massives de sulfate de $\mathrm{Cu}$ (2000 ppm, 4000 ppm et 8000 ppm) ont réduit sensiblement, ou même annulé la survie des plants. Les résultats de l'analyse de la variance ont révélé qu'il n'existe aucune différence significative entre les traitements pour la levée et la reprise. Des résultats similaires sur le taux de levée ont été rapportés par Vignon (2008) sur les variétés de pourghère installées sur les doses croissantes de sulfate de cuivre $\left(\mathrm{CuSO}_{4}\right)$. Par contre, Ahoton et al.(2011) ont enregistré une variabilité des taux de germination allant de 33 à 93\%, lors d'un essai combinant les doses croissantes de sulfate de cuivre $\left(\mathrm{CuSO}_{4}\right)$ et la durée de conservation des semences. Les effets non significatifs de doses croissantes sur le taux moyen de levée des plantes pourraient se justifier par l'utilisation des réserves nutritives de la graine durant la première phase. A 90 jours après levée et transplantation, les meilleurs taux de survie ont été obtenus sur les traitements contaminés à 250 ppm (T1), 500 ppm (T2) et 1000 ppm (T3) comparativement au témoin non contaminé(T0). A la 
dose de 2000 ppm (T4), seuls $50 \%$ de plants repiqués ont survécu ; tandis que la mortalité a été maximale pour les plantes obtenues par semis direct. Par contre, au-delà de 2000ppm, aucune survie n'a été enregistrée pour les 2 essais. Nos résultats vont de paire avec ceux, de Heller (1996) qui a obtenu les taux de survie les plus bas allant de 5 à $50 \%$, par semis direct et entre 79 à $100 \%$, par semis indirect. Andries et Van der Vossen (2007) ont souligné que la meilleure reprise est liée au séjour des plants en pépinière. Alors que les études effectuées par Avila et al.(1949) ont montré que le repiquage peut être réalisé que quand les plants perdent leurs aspects de broussaille, c'est-à-dire quand la coloration passe du vert au gris et quand la formation du bois dans le tronc a eu lieu. La mortalité de plants $J$. curcas a été maximale sur les doses excessives de sulfate de Cu. L'excès de cuivre dans la solution du sol provoque une rhizotoxicité qui s'exprime par une coloration brune des racines, une diminution de la formation des poils absorbants, une diminution de l'élongation racinaire (Kopittke et Menzies, 2006), un épaississement des racines (Marschner, 1995, Panou-Filotheou et Bosabalidis, 2004) et la diminution de la biomasse racinaire (Bravin, 2008) avant d'affecter la croissance des parties aériennes(Marschner, 1995). Les tailles moyennes de plantes de $J$. curcas installées sur les doses inférieures à $2000 \mathrm{ppm}$ ont montré de tailles similaires au traitement témoin (T0) pour les 2 essais. Par contre, la moyenne de hauteur des plantes repiquées, ayant survécu à la dose de 2000 ppm (T4), a été inférieure $(P<0,05)$ par rapport à celles du témoin $(T 0)$ et des doses minimales ( $T, T 2, T 3)$.Les résultats de cette étude ont montré que $\mathrm{J}$. curcas a été capable de se développer dans les pots contaminés à 1000 ppm sans manifester une chlorose au niveau des feuilles et sans une baisse notable de performance (germination et croissance) par rapport au témoin. Pour des espèces moins tolérantes, on note une baisse notable des performances pour des concentrations en $\mathrm{Cu}$ totale inférieures à $300 \mathrm{mg} \mathrm{kg}^{-1}$ (Pederson et al., 2000 ; Brun et al., 2003). Les résultats similaires ont été trouvés par d'autres auteurs notamment, El himer et al. (2013) dans les essais de phytostabilisation de résidus miniers et d'argiles de découvrement par cette même espèce, qui à tolérée une concentration en Cu variant entre 0.45 et 38.8 ppm 26 fois plus inferieur que dans cette étude ; Senou et al. (2012) sur l'essai de phytoextraction du Cu, du Pb et

\section{CONCLUSION}

Le présent travail avait pour objectif d'évaluer la croissance et le potentiel de phytostabilisation de $\mathrm{J}$. curcas sur un substrat contaminé aux doses croissantes du Zn par Vetiveria nigritana et dont la plus forte teneur en Cu était évaluée à $80 \mathrm{mg} \cdot \mathrm{kg}^{-1}$.

Indice biométrique : Pour l'ensemble de traitements, la teneur en Cu retrouvée dans les racines semble élevée par rapport à celles retrouvées dans les parties aériennes de $J$. curcas ; il apparait également que la teneur en $\mathrm{Cu}$ dans les plantes avait augmenté proportionnellement avec les doses de sulfate de cuivre $\left(\mathrm{CuSO}_{4}\right)$ apportées au sol. Cette tendance a déjà été relevée par Piotrowka\&Kabata-Pendia(1997) qui ont souligné que l'absorption du Cu par Jatropha curcas est fonction d'une part de la teneur en Cu dans la solution du sol, et d'autre part de la valeur du $\mathrm{pH}$. Lorsque le $\mathrm{pH}$ est inférieur à 7 , le Cu en solution se trouve majoritairement sous la forme $\mathrm{Cu}^{2+}$, donc très mobile et biodisponible aux plantes. Le calcul de facteur de bioconcentration montre $J$. curcas a absorbé sensiblement le Cu et l'a concentré dans ses racines. Des résultats similaires ont été signalés par les travaux de Qihang et al. (2011) et de El himer et al. (2013) sur la bioaccumulation Co et $\mathrm{Zn}$ dans les racines de Jatropha curcas. Si El himer et al. (2013) attribuent cette différence dans la répartition des ETM entre les racines et les parties aériennes de $\mathrm{J}$. curcas au type de substrat, Marmiroli et al.(2005)précisent que la composition du sol influence la concentration en ETM dans les racines des plantes. Parallèlement, le calcul du facteur de translocation montre que $J$. curcas peut être considéré comme une plante « exclueuse ». Les travaux de Baker \& Walker (1990) et Pauwels (2006) (in Faucon 2009) indiquent que l'aptitude des plantes à absorber les ETM au niveau des tissus racinaires, limite leur transport vers la partie aérienne, ce qui les qualifie des plantes « exclueurs ॥. De tels résultats s'accordent avec les travaux de Yadav et al. (2009) qui ont observé les phénomènes de phytotranslocation des ETM (Zn, As, et Cr)et leur accumulation dans les racines de $J$. curcas. Ces résultats sont intéressants dans le cadre de la phytostabilisation des sols contaminés en $\mathrm{Cu}$, parce que le comportement de J. curcas minimise les risques de transfert d'ETMs vers les autres compartiments de l'Environnement. Non seulement les risques de contamination de la chaîne alimentaire toute caractéristique confèrent d'une part à $J$. curcas la capacité de minimiser le risque de passage des ETM vers les autres compartiments

de Cu. Deux essais expérimentaux ont été installés simultanément en sachets contenant les sols contaminés artificiellement aux doses croissantes allant de 250 à 
8000 ppm de Cu avec les plantes J. curcas obtenus par semis direct et indirect. Les résultats d'analyse statistique montrent que les doses croissantes de Cu apportées au sol n'ont induit aucune différence significative sur la levée et la reprise de J. curcas. En ce qui concerne les paramètres de croissance (taux de survie, nombre des feuilles, taille des plantes et le diamètre au collet des plantes), les doses inférieures à 2000 ppm ont induit des effets similaires au témoin non contaminé pour les deux essais. Par contre, la mortalité a été maximale au-delà de $2000 \mathrm{ppm}$. Néanmoins, la survie a été réduite de moitié pour les individus transplantés avec la dose de 2000 $\mathrm{mg} . \mathrm{kg}^{-1}$ de sulfate de Cu tout en manifestant les signes

\section{BIBLIOGRAPHIE}

Achten, W.M.J., Verchot, L., Frunken, Y.J., Mathijs, E., Singh, V.P. \&Aerts, R., 2008. Jatropha bio-diesel production and use. Biomass \& Bioenergy, 32(12), 1063-1084.

Ahoton, L.E., F. Quenum, G. Mergeai, 2011. Evaluation agromorphologique et sélection des meilleures accessions de pourghère (JatrophacurcasL.) introduites au Bénin. Int. J. Biol. Chem. Sci., 5 (4), 1619-1627.

Alloway B.J., 1995. In "Heavy Metals in Soils", secondeédition, Ed. Alloway B.J., Blackie Academic \& Professional, Londres (RoyameUni).

Alvarenga, P., Gonçalves, A.P., Fernandes, R.M., Varennes, A., Vallini, G., Duarte, E. \& CunhaQueda A.C., 2008. Evaluation of composts and liming materials in the phytostabilization of a mine soil using perennial ryegrass. Science of The Total Environment, 406 (1-2), 43-56.

Andries H, Van der Vossen M., 2007. Oléagineux. In Ressources végétales de l'Afrique tropicale. Vol. 14. PROTA. 260pp.

Avila, J.G., 1949. BrevesInstruçoes para acultura da Purgueira. RepartiçoesTecnica dos serviçosAgricolas, Florestais e Pecuarios., ed. I.N.d.C. Verde. Praia

Baker AJM \& Walker PL., 1990.Ecophysiology of Metal uptake by tolerant plants. In Shaw AJ., 1990. Heavy Metal tolerance in plants: Evolutionary Aspects. 155-177 p.

Bidar G., Garcon G., Pruvot C., Dewaele D., Cazier F., Douay F. \&Shirali P., 2007. Behavior of Trifolium repens and Lolium perenne growing in a heavy metal contaminated field: Plant metal concentration and phytotoxicity. Environmental Pollution, 147, 546-553. de phytotoxicité cuprique. Les calculs de bioaccumulation et translocation, montrent que les plantes de J. curcas sont" exclueuses", du fait que les teneurs en $\mathrm{Cu}$ mesurées dans les racines sont très élevées par rapport à celles observées dans les organes aériens. Ces résultats sont très intéressants dans l'optique de phytostabilisation des sols contaminés principalement en $\mathrm{Cu}$ dans la région du Katanga, du fait que cette faible translocation $J$. curcas minimise non seulement les risques de transfert d' ETMs vers la chaîne alimentaire via les herbivores, mais aussi vers d'autres compartiments de l'Environnement.

Bravin M., 2008. Processus rhizospheriques déterminant la biodisponibilité du cuivre pour le blé dur cultivé en sols à antécédent viticole. Centre International d'Études Supérieures en Sciences Agronomiques, Montpellier SupAgro, $203 \mathrm{p}$.

Brun L.A., Le Corff J. and Maillet J., 2003. Effects of elevated soil copper on phenology, growth and reproduction of five ruderal plant species. Environmental Pollution, 122, 361-368.

Clemens S., 2001. Molecular mechanisms of plant metal tolerance and homeostasis. Planta, 212, 475486.

FAO, 2005.New_LocClim: Local Climate Estimator. FAO Environment and Natural Resources Working Paper, $\mathrm{N}^{\circ} 20$.

Gaston L.A., Locke M.A., Zoblotowicz R.M. \& Reddy K.N., 2001.Spetiali variability of soil propertie and weed population in Mississippi Delta.Soil Science Society of America Journal, 65(2), 449459.

Heller, J., 1996. Physic nut. JatrophacurcasL. Promoting the conservation and use of underutilized and neglected crops Institute of Plant Genetics and Crop Plant Research, Gatersleben/International Plant Genetic Resources Institute, Rome. 1, 1$66 \mathrm{p}$.

KabataPendias A., 2010. Trace elements in soils and plants. 3rd Edition, CRC Press, Boca Raton, Florida, $432 \mathrm{p}$.

Kabata-Pendias A. \&Pendias H., 1992. Trace elements in soils and plants. 2nd Edition, CRC Press, Boca Raton, Florida, 365p.

Kabata-Pendias, A., Pendias, H., 2001. Trace Elements in Soils and Plants. CRC Press, Boca Ratón, 413p.

Kopittke P.M. \&Menzies N.W., 2006.Effect of Cu toxicity on growth of cowpea (Vigna unguiculata). Plant 
and Soil, 279, 287-296.

Kumar, A., \& Sharma, S., 2008. An evaluation of multipurpose oil seed crop for industrial uses (JatrophacurcasL.) : A review. IndustrialCrops and Products, 28, 1-10.

Malaisse F., 1997. Se nourrir en forêt claire africaine. Approche écologique et nutritionnelle. Centre Les presses Agronomiques de Gembloux, ASBL. Gembloux.1665/3.

Marchiol L., Sacco P., Assolari S. \&Zerbi G., 2004.Reclamation of polluted soil: Phytoremediation potential of corp-related Brassica species. Water, Air, SoilPollut. 185, 345-356.

Marmiroli M., Antonioli G., Maestri E. \&Marmiroli N., 2005. Evidence of the involvement of plant lingocellulosic structure in the sequestration of $\mathrm{Pb}$ : an Xray spectroscopy-based analysis. EnvironmentalPollution 134: 217-227.

Marschner H., 1995. Mineral nutrition of higher plants. Second Edition, Academic Press, $889 \mathrm{p}$.

McGrath, S.P., 1998. Phytoextraction for soil remediation. Plants that hyperaccumulate heavy metals. R.R. Brooks. Wallingford, CAB International, p. 261287.

Mench, M., Vangronsveld, J., Lepp, N., Bleeker, P., Ruttens, A. \&Geebelen, W., 2005. Phytostabilisation of metal - contaminated sites.in T. N. Springer Editor Phytoremediation of metal - contaminated soils, Echevarria, G., Morel, J. L., Goncharova, N. (Eds), Trest, Czech Republic, p. 109-190.

Mpundu M.M., 2010. Contaminations des sols en éléments traces métalliques à Lubumbashi (Katanga RDC). Évaluation des risques de contamination de la chaine alimentaire et choix des solutions de remediation. Thèse de doctorat, Université de Lubumbashi, 401p.

Mujinya B.B., Mees F., Boeckx P., Bode S., Baert G., Erens H., Delefortrie S., Verdoodt A., Ngongo M.L., Van Ranst E., 2011. The origin of carbonate in the termite mounds of the Lubumbashi area, DR Congo. Geoderma, 165, 95-105

Neff C. \& Scheid A., 2008. Les Biocarburants: Analysent du potentiel de production de biocarburants à l'échelle internationale et en Tunisie. Etude GTZ. Université de Karlsruhe, 29 p.
Ngoy S.M., 2010. Phytostabilisation des sols contaminés en métaux lourds par l'activité minière au Katanga " Cas du quartier Gécamines/PengaPenga contaminés en métaux par les émissions de la fonderie de cuivre de I'Usine Gécamines/Lubumbashi ». Thèse de doctorat. Universié de Lubumbashi. $10 \mathrm{p}$.

Panou-Filotheou, \&Bosabalidis A.M., 2004 Root structural aspects associated with copper toxicity in oregano (Origanum vulgare subsp. Hirtum). Plant science, 166, 1497-1504.

Pederson MB, Kjær C and Elmegaard N., 2000.Toxicity and accumulation in black Bindweed (Fallopia convolvulus) in relation to bioavailability and age of soil contamination.Archives in Environmental Contamination and Toxicology, 39, 431- 439.

Pellet J. D. \& Pellet E., 2007. Jatropha curcas, le meilleur des biocarburants : mode d'emploi, histoire et avenir d'une plante extraordinaire. Lausanne (Favre). $1 \mathrm{p}$.

Senou I., Gnankambary Z., Some A. N. \&Sedogo M. P., 2013. Phytoextraction du cadmium, du cuivre, du plomb et du zinc par Vetiverianigritanaen sols ferrugineux tropicaux et en sols vertiques au Burkina Faso (Afrique de l'ouest). Int. J. Biol. Chem. Sci. 6(4), 1437-1452.

Useni Sikuzani, Mwamba llunga, Mwamba Mulembo, Ntumba Katombe, Lwalaba Wa Lwalaba, Assani Bin Lukangila, KanyengaLubobo \& Baboy Longanza, 2014. Amélioration de la qualité des sols acides de Lubumbashi (Katanga, RD Congo) par l'application de différents niveaux de compost de fumiers de poules. Journal of Applied Biosciences, 77:6523 - 6533.

Vignon, C.M.R., 2008. Étude des techniques culturales de Jatropha curcas: cas des plantations expérimentales du SET UP dans la commune de Zagnanado, Mémoire de fin de formation pour l'obtention du diplôme de licence en agronomie à l'Université Africaine de Technologie et de Management (UATM- GASA Formation), Cotonou, Bénin, $40 \mathrm{p}$.

Vranken I., Amisi M.Y., Munyemba K.F., Bamba I., Veroustraete F., Visser M., Bogaert J., 2013. The spatial footprint of the non-ferrous mining industry in Lubumbashi. Tropicultura, 1, 20-27 